\title{
Fungal co-infection in COVID-19 patients: evidence from a systematic review and meta-analysis
}

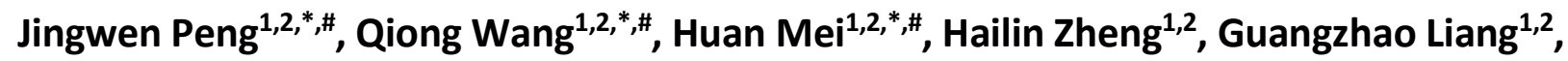 \\ Xiaodong She ${ }^{1,2}$, Weida Liu ${ }^{1,2,3}$ \\ ${ }^{1}$ Department of Medical Mycology, Institute of Dermatology, Chinese Academy of Medical Science and Peking \\ Union Medical College, Nanjing 210042, Jiangsu, China \\ 2Jiangsu Key Laboratory of Molecular Biology for Skin Diseases and STIs, Nanjing 210042, Jiangsu, China \\ ${ }^{3}$ Center for Global Health, School of Public Health, Nanjing Medical University, Nanjing 211166, China \\ *Equal contribution \\ ${ }^{\#}$ Co- first authors
}

Correspondence to: Weida Liu; email: liuwd@pumcderm.cams.cn

Keywords: fungal co-infection, COVID-19, meta-analysis, systematic review

Received: December 11, $2020 \quad$ Accepted: February 18, $2021 \quad$ Published: March 19, 2021

Copyright: (C) 2021 Peng et al. This is an open access article distributed under the terms of the Creative Commons Attribution License (CC BY 3.0), which permits unrestricted use, distribution, and reproduction in any medium, provided the original author and source are credited.

\section{ABSTRACT}

Coronavirus disease 2019 (COVID-19) has infected tens of millions of people worldwide within the last year. However, the incidence of fungal co-infection in COVID-19 patients remains unclear. To investigate the association between fungal co-infection and mortality due to COVID-19, we systematically searched Medline, Embase, MedRxiv and Cochrane Library for eligible studies published in the period from 1 January to 1 December 2020. We performed a meta-analysis of nine studies that met the inclusion criteria. In total, data from 2780 patients and $\mathbf{4 2 6}$ patients were included who were admitted to the ICU. In eight of the articles, 211 participants died due to COVID-19 infection, which means an overall mortality rate of $10.9 \%$. The overall pooled proportion of fungal co-infection in COVID-19 patients was $0.12(95 \% \mathrm{Cl}=0.07-0.16, \mathrm{n}=$ 2780, $\left.I^{2}=96.8 \%\right)$. In terms of mortality in COVID-19 patients with fungal infection, the overall pooled proportion of mortality was $0.17\left(95 \% \mathrm{Cl}=0.10-0.24, \mathrm{n}=1944, I^{2}=95.6 \%\right)$. These findings provide evidence suggesting a favorable use for empirical antibiotics in the majority of patients when COVID-19 infection is diagnosed. Our analysis is investigating the use of antifungal therapy to treat COVID-19 can serve as a comprehensive reference for COVID-19 treatment.

\section{INTRODUCTION}

The recent outbreak of coronavirus disease 2019 (COVID-19), a new disease mostly manifested as viral pneumonia, started as a local epidemic but developed within a few months into a worldwide pandemic with high morbidity and mortality rates [1-3]. However, breakthroughs in the development of specific therapeutic agents are still needed and newly developed vaccines against COVID-19 infection are available only in small quantity. Thus, the most effective way to address the pandemic is the prevention of further infection. This is achieved by using specific strategies such as early diagnosis and subsequent quarantine, and policies preventing gatherings, such as enforced social distancing. From the previous coronavirus outbreak of severe acute respiratory syndrome (SARS), we found that fungal co-infection of coronavirus patients could significantly increase mortality rates [4-7]. The significance of fungal co-infection in COVID-19 patients, however, especially in patients with severe and critical conditions, is still poorly understood.

As with other respiratory diseases such as influenza, where approximately $25 \%$ of elderly patients acquire secondary co-infections, a similar co-infection has also 
been seen in COVID-19 patients [6]. However, data remain limited regarding the impact of fungal coinfection and associated clinical outcomes. In many studies looking at the treatment of COVID-19 patients, the empirical use of antibiotics for the majority of patients is known [6-9]. However, there is evidence that inflammatory serological markers, which are usually associated with bacterial infection, such as raised procalcitonin and C-reactive protein, may appear in patients with COVID-19 who have no bacterial coinfection. Paradoxically, the administration of antibiotics prevents co-infection, which has been associated with a more severe clinical condition in COVID-19 patients [10-12]. Therefore, there is a clinical demand for a robust investigation into the role of co-infection in patients with COVID-19.

In this study, we performed a systematic review and meta-analysis of nine studies investigating fungal coinfection in COVID-19 patients, to estimate the association between fungal co-infection and mortality. These findings may help to inform future clinical management and treatment of COVID-19 patients. In the context of rising levels of antimicrobial resistance, we aim to enable a sustainable and judicious antibiotic administration.

\section{RESULTS}

\section{Study selection and characteristics}

The combined search terms yielded 273 articles and a primary review of the titles and abstracts identified 32 articles that warranted a full manuscript review. After screening the literature based on the inclusion criteria, nine articles were identified as potentially relevant articles (Figure 1). In total, nine studies including 2780 participants and 426 patients admitted to the ICU met the inclusion criteria and were selected for metaanalysis. Of the participants, there was 211 death reported in eight of the articles, for an overall mortality rate of $10.9 \%$. In one article, the outcomes were not reported. Four (44\%) of the included studies were from China, two were from the United Kingdom (22\%), one (11\%) was from Pakistan, and one $(11 \%)$ was from Spain. The characteristics of the selected studies are summarized in Table 1.

For fungal co-infection in COVID-19 patients, the overall pooled proportion of patients who had laboratory-confirmed fungal co-infection was 0.12 $[(95 \%$ confidence intervals $(\mathrm{CI})=0.07-0.16, \mathrm{n}=2780$, $\left.\left.I^{2}=96.8 \%\right)\right]$. Subgroup analysis from studies with separate data for Asian patients showed that a greater proportion of them had fungal co-infections when compared to the patients in the studies from the U.K. and Spain $(0.15,95 \% \mathrm{CI}=0.03-0.27), \mathrm{n}=679, I^{2}=$ $95.5 \%$ versus $0.07,95 \% \mathrm{CI}=0.03-0.10, \mathrm{n}=2101, l^{2}=$ $95.4 \%$ ). Subgroup analyses were not significantly different for the severity of disease, study type, or test method. Sensitivity analysis excluding one study, did not significantly affect the overall proportion of patients with fungal co-infection, nor did it decrease the heterogeneity $\left(0.14,95 \% \mathrm{CI}=0.06-0.21, I^{2}=96.0 \%\right)$. There were no data from the remaining studies relating to the time from admission to detection of co-infection. Specific data are summarized in Figure 2, Table 2, and Supplementary Figure 1.

We also analyzed Aspergillus co-infection in COVID19 patients, whereby the overall pooled proportion of patients was $0.06\left(95 \% \mathrm{CI}=0.04-0.08, \mathrm{n}=2780, I^{2}=\right.$ 96.0\%). The difference in proportions between Asian and Caucasian patients was significant $(0.13,95 \% \mathrm{CI}=$ $0.00-0.25, \mathrm{n}=673, I^{2}=97.2 \%$ and $0.01,95 \% \mathrm{CI}=$ $0.00-0.03, \mathrm{n}=2107, I^{2}=89.9 \%$ respectively). This correlation was not observed in other subgroups, such as race, study type or test method. However, we also failed to find a significantly different proportion between patients in ICU and the mixed hospitalized population (0.06 and 0.07). Specific data are summarized in Figure 3, Table 3 and Supplementary Figure 2.

In terms of patient mortality with COVID-19 combined with fungal infection, we found that the overall pooled proportion of mortality was 0.17 (95\% CI $=0.10-0.24$, $\mathrm{n}=1944, I^{2}=95.6 \%$ ). Subgroup analysis shown a significant difference in the proportions between patients in ICU and the mixed hospitalized population $\left(0.36,95 \% \mathrm{CI}=0.09-0.63, \mathrm{n}=286, I^{2}=95.4 \%\right.$ and $0.0695 \% \mathrm{CI}=0.02-0.10, \mathrm{n}=1658, I^{2}=84.6 \%$ respectively). We also found significantly different proportions in the case size of the subgroups (proportion in case size of the $<200$ subgroup was $0.23,95 \% \mathrm{CI}=$ $0.07-0.40, \mathrm{n}=462, I^{2}=96.9 \%$ and proportion in case size of the $>200$ subgroup was $0.08,95 \% \mathrm{CI}=0.04$ $0.12, \mathrm{n}=1482, I^{2}=78.3 \%$ ). This correlation was not observed in other subgroups, such as race, study type, test method, rate of Aspergillus and rate of fungal infection. Specific data are described in Figure 4, Table 4 and Supplementary Figure 3.

\section{Publication bias and sensitivity analysis}

Begg's funnel plot and Egger's test were performed to assess publication bias. We additionally conducted sensitivity analyses by omitting one study at a time in the calculation of a summary outcome (Supplementary Figures 1-3). Although the sample sizes for cases in all eligible studies varied, corresponding pooled proportions and 95\% CIs were not qualitatively altered 
regardless of the study size. No other single study influenced pooled proportion and 95\% CI qualitatively.

\section{DISCUSSION}

The World Health Organization (WHO) declared COVID-19 as a public health emergency of international concern in February 2020 and since then, it has developed into a worldwide pandemic associated with substantial morbidity and mortality. At the time of writing, over 99 million laboratory-diagnosed cases of
COVID-19 infections have been reported in 212 countries, contributing to over $2,100,000$ death.

Antimicrobial therapy has a role in the treatment of suspected or confirmed bacterial or fungal respiratory co-infection [13]. This may be empirical or targeted in patients presenting at hospital, or for the management of nosocomial infection acquired during admission to hospital, such as hospital-acquired pneumonia or ventilator-associated pneumonia [14]. Patients may also be suffering from secondary co-infections not linked to

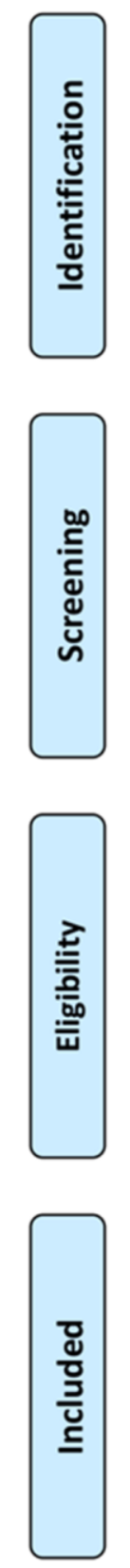

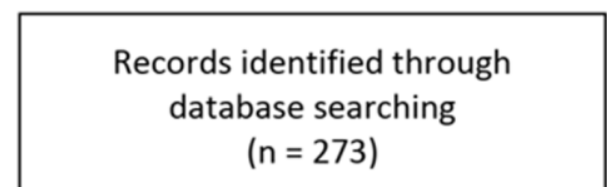

$(n=273)$

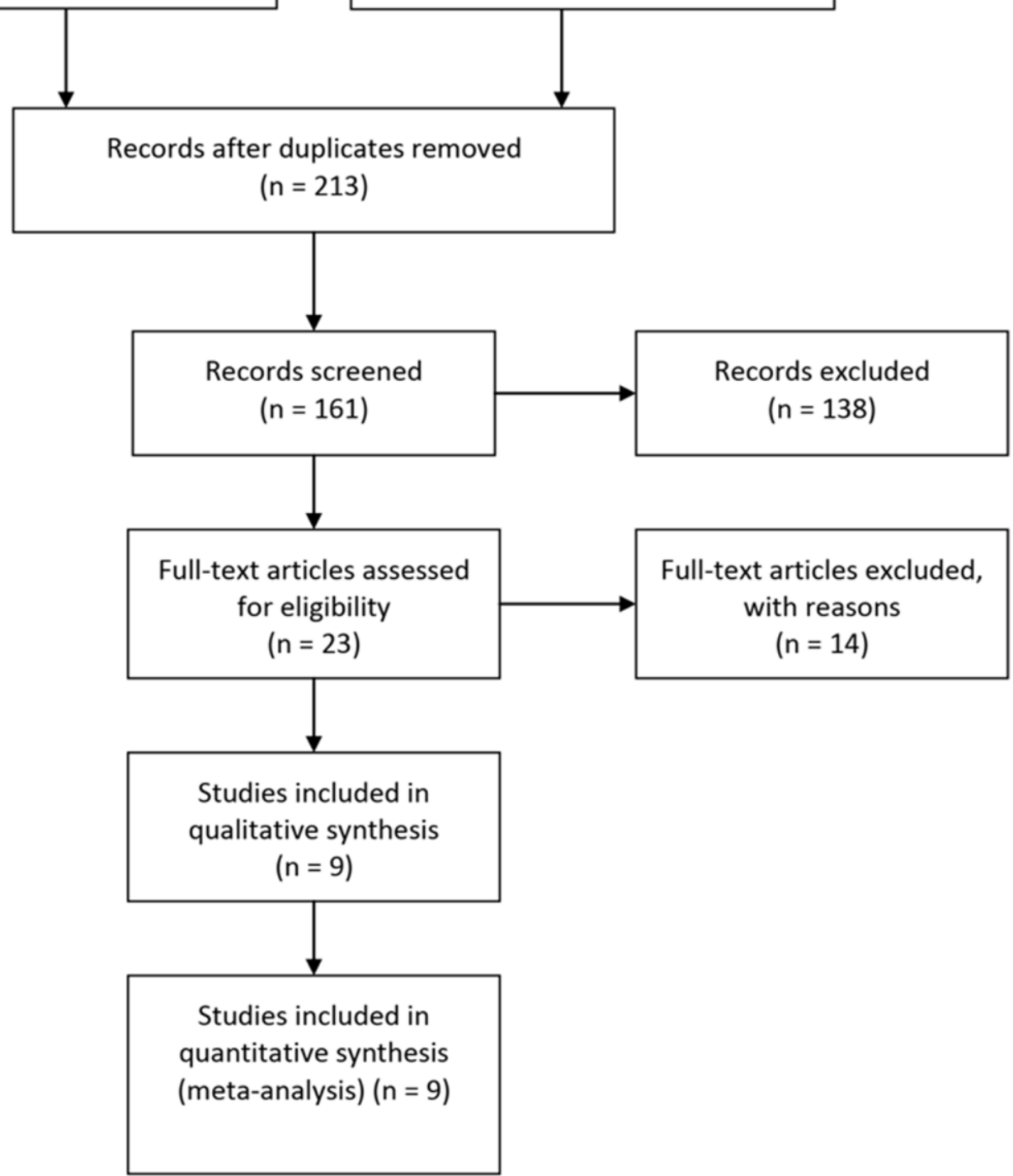

Additional records identified through other sources $(n=256)$ 
Table 1. Characteristics of all studies describing fungal co-infections in the meta-analysis.

\begin{tabular}{|c|c|c|c|c|c|c|c|c|c|c|}
\hline Author & Country & Race & Severity & Study type & Method & Total & ICU & Deaths (\%) & Fungal $(\%)$ & $\begin{array}{c}\text { Aspergillus } \\
(\%)\end{array}$ \\
\hline Yang [5] & China & Asian & ICU & Case series & Culture & 52 & 52 & $32(61.5 \%)$ & $3(5.8 \%)$ & $2(3.8 \%)$ \\
\hline Wang [13] & China & Asian & Mix & Case series & Culture & 29 & & $2(6.9 \%)$ & $2(6.9 \%)$ & $0(0 \%)$ \\
\hline Chen [19] & China & Asian & ICU & Case series & Culture & 99 & 23 & $11(11.1 \%)$ & $4(4.0 \%)$ & $1(1.0 \%)$ \\
\hline Zhu [10] & China & Asian & Mix & Retrospective study & RT-PCR & 257 & 3 & $0(0 \%)$ & $60(23.3 \%)$ & $66(25.7 \%)$ \\
\hline White [11] & UK & Caucasian & ICU & Prospective study & Mix & 135 & 135 & $51(37.8 \%)$ & $36(26.7 \%)$ & $19(14.1 \%)$ \\
\hline $\begin{array}{l}\text { Garcia-Vidal } \\
\text { [16] }\end{array}$ & Spain & Caucasian & Mix & Prospective study & Culture & 989 & 146 & $97(9.8 \%)$ & $7(0.7 \%)$ & $3(0.3 \%)$ \\
\hline
\end{tabular}

Mix of Severity, Symptoms of the disease include moderate, severe, and critical; Mix of the Method, Detection methods include culture, serological diagnosis, and reverse transcription-polymerase chain reaction (RT-PCR); ICU, intensive-care unit; UK, United Kingdom.

their respiratory presentation, for example urinary tract or blood infections. Therefore, empirical treatment with antimicrobials is reasonable for bacterial/fungal pneumonia in unwell patients. Some national guidelines and evidence from cases have suggested the use of broad-spectrum antibiotics or the benefit of typical antibiotic cover.

Early studies have reported that patients with severe viral infection tend to get infections with Aspergillus,

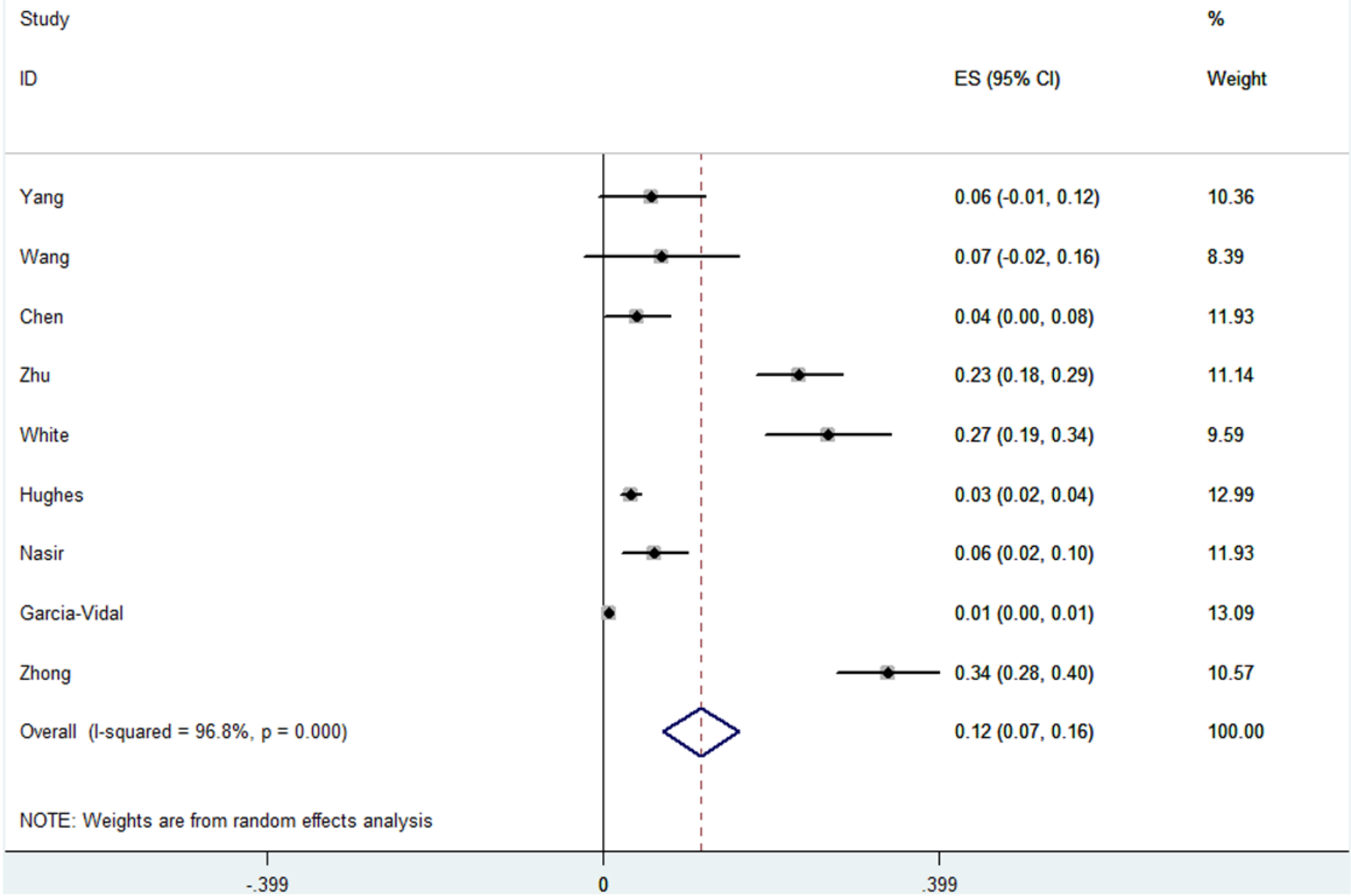

Figure 2. Forest plot of fungal infection in COVID-19 patients. 
Table 2. Proportion of fungal co-infections and COVID-19.

\begin{tabular}{lcccc}
\hline & Proportion(95\% CI) & $\boldsymbol{P}$ & $\boldsymbol{P}_{\boldsymbol{h}}$ & $\boldsymbol{I}^{\mathbf{2} \%}$ \\
\hline Total & $0.12(0.07-0.16)$ & 0.000 & 0.000 & $96.8 \%$ \\
Race & & & & \\
Asian & $0.15(0.03-0.27)$ & 0.015 & 0.000 & $95.5 \%$ \\
Caucasian & $0.07(0.03-0.10)$ & 0.001 & 0.000 & $95.4 \%$ \\
Severity & & & & \\
ICU & $0.12(-0.01-0.24)$ & 0.065 & 0.000 & $93.0 \%$ \\
MIX & $0.12(0.06-0.17)$ & 0.000 & 0.000 & $97.5 \%$ \\
Study type & & & & \\
Case series & $0.05(0.02-0.08)$ & 0.003 & 0.804 & $0.0 \%$ \\
Retrospective study & $0.16(0.04-0.29)$ & 0.010 & 0.000 & $97.9 \%$ \\
Prospective study & $0.13(-0.12-0.39)$ & 0.301 & 0.000 & $97.8 \%$ \\
Method & & & & \\
Culture & $0.04(0.01-0.07)$ & 0.016 & 0.008 & $71.2 \%$ \\
RT-PCR & $0.23(0.18-0.29)$ & 0.000 & - & - \\
MIX & $0.15(-0.08-0.38)$ & 0.211 & 0.000 & $97.3 \%$ \\
Fungus chip & $0.34(0.28-0.40)$ & 0.000 & - & - \\
Case size & & & & \\
$>200$ & $0.14(0.08-0.20)$ & 0.000 & 0.000 & $98.5 \%$ \\
$<200$ & $0.10(0.03-0.16)$ & 0.003 & 0.804 & $0.0 \%$ \\
\hline
\end{tabular}

Mix of Severity, Symptoms of the disease include moderate, severe and critical; Mix of the Method, Detection methods include culture, serological diagnosis, reverse transcription-polymerase chain reaction; (RT-PCR); ICU, intensive-care unit; $\mathrm{Cl}$, confidence interval; $P_{\mathrm{h}}, P$ value of heterogeneity, $P$ value of $\mathrm{Q}$-test for the heterogeneity test; $I^{2}, 0-25$, no heterogeneity; $25-50$, modest heterogeneity; 50 , high heterogeneity.

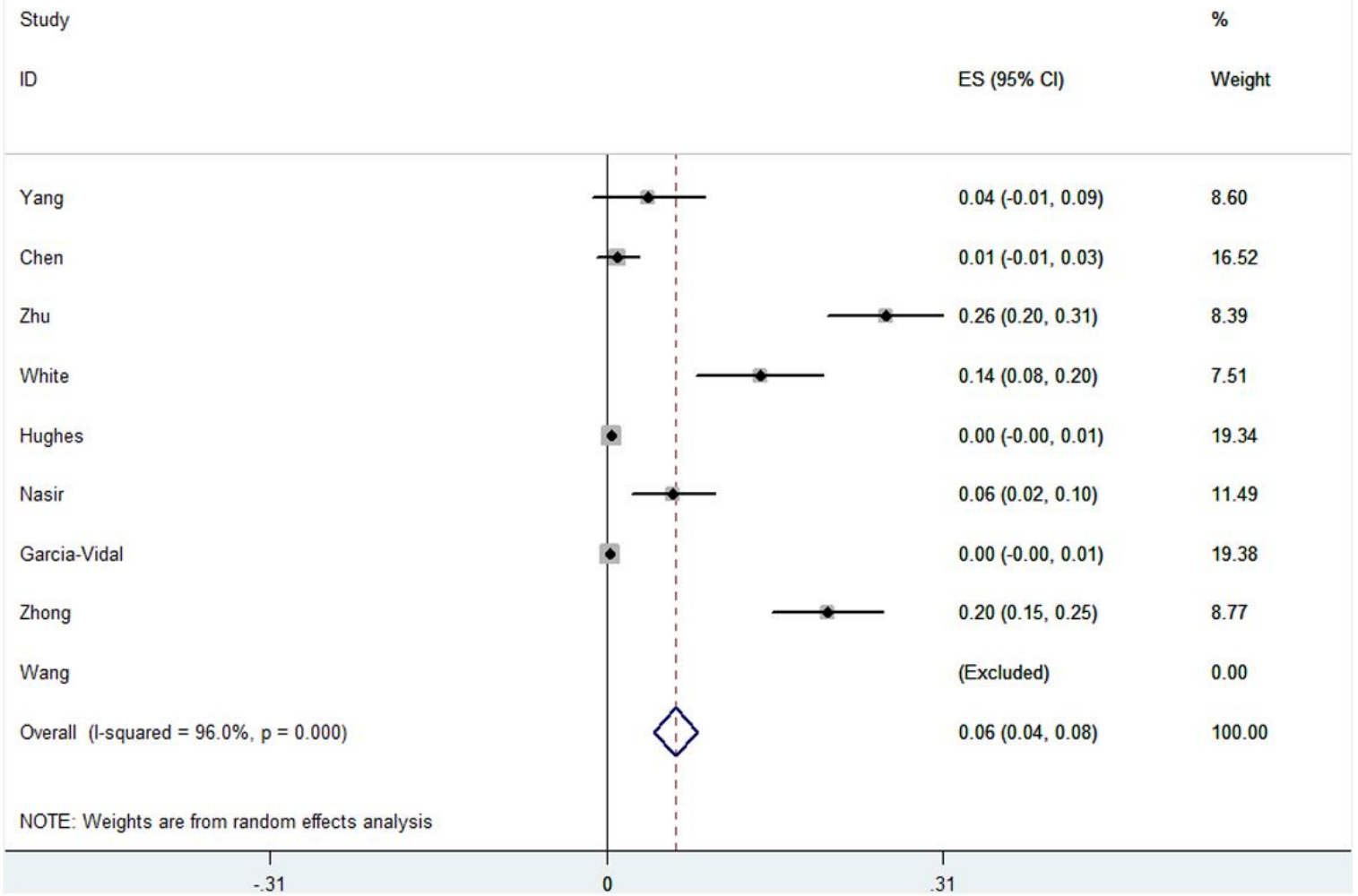

Figure 3. Forest plot of aspergillus infection in COVID-19 patients. 
Table 3. Proportion of Aspergillus co-infections and COVID-19.

\begin{tabular}{lcccc}
\hline & Proportion(95\%CI) & $\boldsymbol{P}$ & $\boldsymbol{P}_{\boldsymbol{h}}$ & $\mathbf{I}^{\mathbf{2} \%}$ \\
\hline Total & $0.06(0.04-0.08)$ & 0.000 & 0.000 & $96.0 \%$ \\
Race & & & & \\
Asian & $0.13(0.00-0.25)$ & 0.046 & 0.000 & $97.2 \%$ \\
Caucasian & $0.01(0.00-0.03)$ & 0.028 & 0.000 & $89.9 \%$ \\
Severity & & & & \\
ICU & $0.06(-0.01-0.13)$ & 0.111 & 0.000 & $88.5 \%$ \\
MIX & $0.07(0.05-0.09)$ & 0.000 & 0.000 & $97.4 \%$ \\
Study type & & & & \\
Case series & $0.01(-0.00-0.03)$ & 0.147 & 0.320 & $0.0 \%$ \\
Retrospective study & $0.13(0.01-0.25)$ & 0.031 & 0.000 & $98.0 \%$ \\
Prospective study & $0.07(-0.07-0.20)$ & 0.318 & 0.000 & $95.3 \%$ \\
Method & & & & \\
Culture & $0.02(-0.00-0.04)$ & 0.073 & 0.013 & $72.0 \%$ \\
RT-PCR & $0.26(0.20-0.31)$ & 0.000 & - & - \\
MIX & $0.07(-0.07-0.20)$ & 0.314 & 0.000 & $95.2 \%$ \\
Fungus chip & $0.20(0.15-0.25)$ & 0.000 & - & - \\
Case size & & & \\
$>200$ & $0.03(-0.00-0.07)$ & 0.062 & 0.057 & $65.0 \%$ \\
$<200$ & $0.08(0.06-0.11)$ & 0.000 & 0.000 & $97.6 \%$ \\
\hline
\end{tabular}

Mix of Severity, Symptoms of the disease include moderate, severe and critical; Mix of the Method, Detection methods include culture, serological diagnosis, and reverse transcription-polymerase chain reaction (RT-PCR); ICU, intensive-care unit; $\mathrm{Cl}$, confidence interval; $P_{\mathrm{h}}, P$ value of heterogeneity, $P$ value of $Q$-test for the heterogeneity test; $I^{2}$, $0-25$, no heterogeneity; $25-50$, modest heterogeneity; 50 , high heterogeneity.

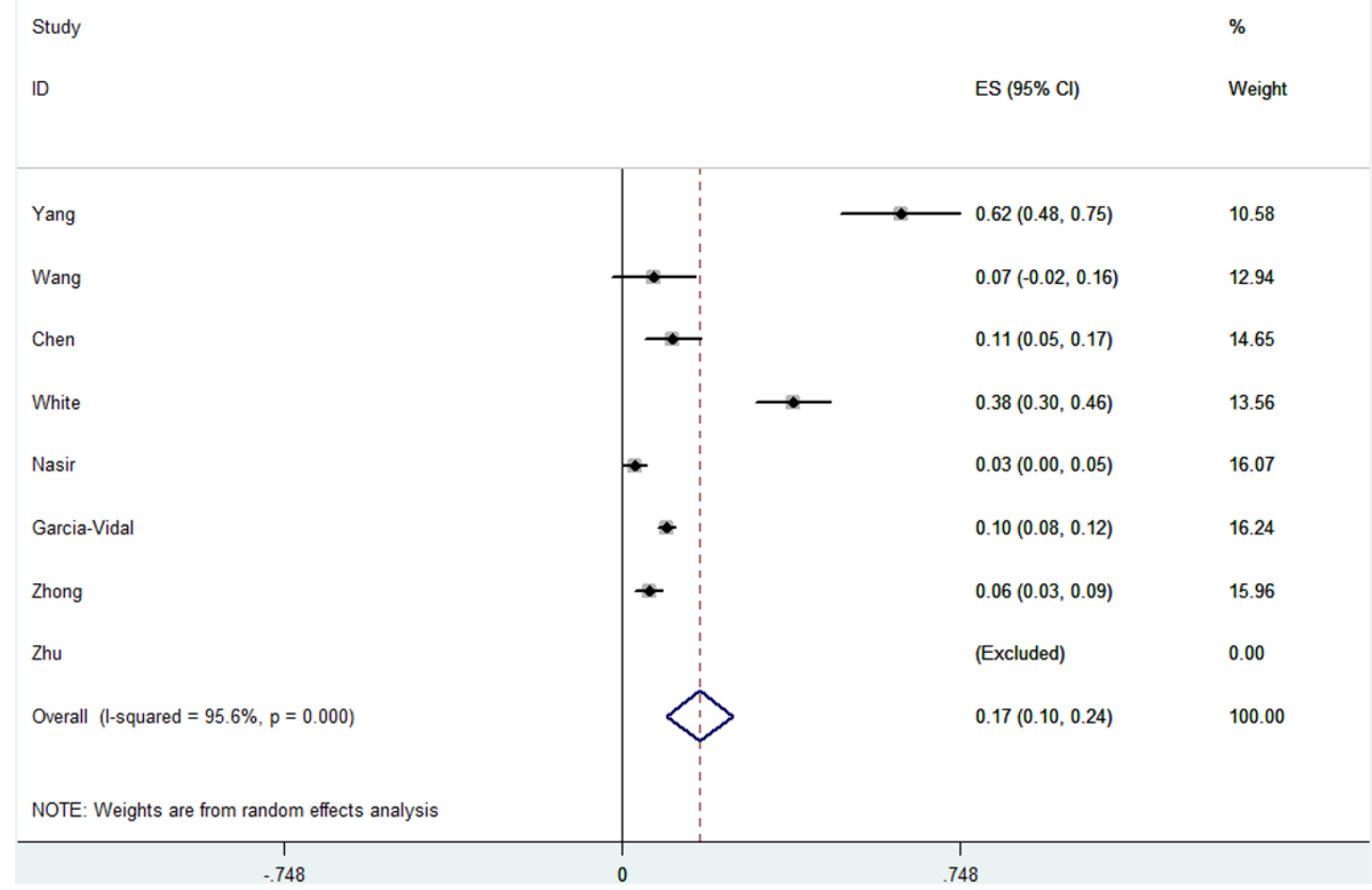

Figure 4. Forest plot of mortality in COVID-19 patients with fungal co-infections. 
Table 4. Proportion of mortality in fungal co-infections and COVID-19.

\begin{tabular}{|c|c|c|c|c|}
\hline & $\operatorname{Proportion}(95 \% \mathrm{CI})$ & $\boldsymbol{P}$ & $P_{h}$ & $I^{2} \%$ \\
\hline Total & $0.17(0.10-0.24)$ & 0.000 & 0.000 & $95.6 \%$ \\
\hline \multicolumn{5}{|l|}{ Race } \\
\hline Asian & $0.20(0.05-0.36)$ & 0.012 & 0.000 & $95.4 \%$ \\
\hline Caucasian & $0.16(0.05-0.26)$ & 0.004 & 0.000 & $97.1 \%$ \\
\hline \multicolumn{5}{|l|}{ Severity } \\
\hline ICU & $0.36(0.09-0.63)$ & 0.008 & 0.000 & $96.5 \%$ \\
\hline MIX & $0.06(0.02-0.10)$ & 0.002 & 0.000 & $84.6 \%$ \\
\hline \multicolumn{5}{|l|}{ Study type } \\
\hline Case series & $0.26(-0.01-0.53)$ & 0.058 & 0.000 & $96.1 \%$ \\
\hline Retrospective study & $0.04(0.01-0.07)$ & 0.008 & 0.116 & $59.6 \%$ \\
\hline Prospective study & $0.23(-0.04-0.51)$ & 0.093 & 0.000 & $97.7 \%$ \\
\hline \multicolumn{5}{|l|}{ Method } \\
\hline Culture & $0.16(0.07-0.24)$ & 0.000 & 0.000 & $95.2 \%$ \\
\hline MIX & $0.38(0.30-0.46)$ & 0.000 & - & - \\
\hline Fungus chip & $0.06(0.03-0.09)$ & 0.000 & - & - \\
\hline \multicolumn{5}{|l|}{ Case size } \\
\hline$>200$ & $0.08(0.04-0.12)$ & 0.000 & 0.032 & $78.3 \%$ \\
\hline$<200$ & $0.23(0.07-0.40)$ & 0.006 & 0.000 & $9.9 \%$ \\
\hline \multicolumn{5}{|l|}{ Rate of Aspergillus } \\
\hline$<5 \%$ & $0.18(0.08-0.28)$ & 0.000 & 0.000 & $96.4 \%$ \\
\hline$>5 \%$ & $0.17(-0.03-0.36)$ & 0.093 & 0.000 & $96.1 \%$ \\
\hline \multicolumn{5}{|l|}{ Rate of fungal } \\
\hline$<10 \%$ & $0.16(0.07-0.24)$ & 0.006 & 0.000 & $95.2 \%$ \\
\hline$>10 \%$ & $0.20(-0.10-0.53)$ & 0.175 & 0.000 & $98.0 \%$ \\
\hline
\end{tabular}

Mix of Severity, Symptoms of the disease include moderate, severe and critical; Mix of the Method, Detection methods include culture, serological diagnosis, and, reverse transcription-polymerase chain reaction; (RT-PCR), ICU, intensive-care unit; $\mathrm{Cl}$, confidence interval; $P_{\mathrm{h}}, P$ value of heterogeneity, $P$ value of $Q$-test for the heterogeneity test; $l^{2}$, $0-25$, no heterogeneity; $25-50$, modest heterogeneity; 50 , high heterogeneity.

Candida, Cryptococcus neoformans, Pneumocystis, or other fungal species, and that this has an apparent association with increased morbidity and mortality [15-18]. Chen et al. [19] firstly described fungal infection in COVID-19 patients whereby the incidence was 4\%, higher than that seen for bacterial infections, at only $1 \%$. Since then, several studies have investigated the prevalence of fungal infections in patients with COVID-19. There are also an increasing number of reports from Europe, where patients with COVID-19 were associated with pulmonary aspergillosis $[5,11,13]$. In patients with acute respiratory distress syndrome caused by COVID-19, an increased risk of microbial infections has been reported, even in the absence of predisposing immunocompromising conditions. Epidemiological and clinical characteristics have been described in earlier research and some researchers have suggested that patients should be routinely screened for bacterial and fungal infection after a confirmation of COVID19 infection $[10,11]$.

In this study, the overall pooled proportion of fungal infections in COVID-19 patients was $0.12,95 \% \mathrm{CI}=$ $0.07-0.16, I^{2}=96.8 \%$. Subgroup analysis of studies with separate data for Asian patients, showed that a greater proportion of them had fungal co-infections when compared to patients from non-Asian studies (0.15 versus 0.07$)$. We also analyzed Aspergillus coinfection in COVID-19 patients and the overall pooled 
proportion of patients was $0.06(95 \% \mathrm{CI}=0.04-0.08$, $\left.I^{2}=96.0 \%\right)$. Results of subgroup analysis were similar to fungal infection with COVID-19 patients. A likely explanation is that many physicians did not associate the combination of COVID-19 and fungal infection in the early stages of COVID-19, due to the urgency of the epidemic, which is an important reason for the confounding factors associated with patient illness. We suggest that all physicians should be more attentive to mycological diagnoses in COVID-19 patients at early stages of infection, to reduce the risk of this progressing to a critical illness.

In terms of mortality of COVID-19 patients combined with fungal infection, we found that the overall pooled proportion of mortality was $0.17(95 \% \mathrm{CI}=0.10-0.24$, $I^{2}=95.6 \%$ ). Subgroup analysis showed a significant difference in proportions between patients in ICU and the mixed hospitalized population (0.36 versus 0.06 ). We also found significantly different proportions in the case size subgroup (proportion in case size of $<200$ subgroup was $0.23,95 \% \mathrm{CI}=0.07-0.40, I^{2}=96.9 \%$ and proportion in case size of $>200$ subgroup was $0.08,95 \%$ $\left.\mathrm{CI}=0.04-0.12, I^{2}=78.3 \%\right)$. This correlation was not observed in the rate of Aspergillus subgroup or rate of fungal subgroup. A potential explanation for this is that several physicians may not think that mycology detection is necessary for many terminally ill patients. In addition, mycological detection is also not validated for upper respiratory tract specimens in COVID-19 patients. In light of the current diagnostic difficulties and uncertainties relating to the risks associated with fungal infection in COVID-19 pneumonia, clinicians should maintain a high level of diligence for this infection in critically ill patients.

Some limitations of the current study need to be addressed [20]. First, only nine studies were examined, and the relatively small total sample size had limited power for the exploration of real associations. Second, subgroup analyses involved relatively small groups, which may not impart sufficient statistical power to explore the real association and are more likely to reveal greater beneficial effects than large-scale trials. Third, every physician has a different treatment for clinical diagnostic and treatment algorithms, which would allow for adjustments by other factors. In addition, inclusion of zero-event trials can sometimes decrease the effect size estimate and narrow confidence intervals.

\section{CONCLUSIONS}

To the best of our knowledge, this is the only systematic review and meta-analysis investigating the proportions of fungal infection of COVID-19 patients within a large sample size. We summarized all available studies for an overall pooled proportion of fungal co-infection in COVID-19 patients. Overall, these findings provide evidence favoring a thoughtful empirical administration of antibiotics for the vast majority of patients with COVID-19 infection. Our analysis of the effects of utilizing antifungal therapy to treat COVID-19 patients can serve as a comprehensive reference for future COVID-19 treatment.

\section{MATERIALS AND METHODS}

\section{Study selection}

We searched Medline, Embase, MedRxiv and Cochrane Library with the search terms co-infection, coronavirus, severe acute respiratory syndrome coronavirus 2, SARS-CoV-2, 2019-nCoV and COVID-19 for studies published from January 1, 2020 up to December 1, 2020 , and we manually searched the references of the selected articles for additional relevant articles (Figure 1). As this study was a meta-analysis, it did not require ethical approval.

\section{Data extraction and verification}

Information pertaining to the enrolled studies is listed in Table 1, including: (I) the author's name, (II) country or region of origin, (III) ethnicity or race, (IV) severity of illness, (V) study type, (VI) method, (VII) number of total patients, (VIII) number of patients in ICU, (IX) number of deaths, $(\mathrm{X})$ fungal co-infection and (XI) Aspergillus co-infection. First, three authors (Jingwen Peng, Qiong Wang and Huan Mei) independently screened the citations for articles meeting our inclusion criteria and extracted all of the data. If at least two of them agreed, the study was included in the metaanalysis. The data included in the study were presented in the form of lists and differences were considered and identified in the quality evaluation. Next, everyone extracted while the other cross-checked the data. Disagreements were resolved by reviewing and discussion.

\section{Statistical analyses}

The statistical significance of the pooled proportion was determined with the Z-test, and $P$-values $<0.05$ were considered as statistically significant. Data were pooled from the meta-analysis with the random-effects model using the DerSimonian and Laird method, and the fixedeffects model using the Mantel-Haenszel method. To evaluate the influence of individual data sets by overall pooled proportion, we conducted forest plot analysis to determine the stability of our results. We also carried out sensitivity analysis in which a single study within the 
overall meta-analysis was deleted one at a time. We applied Funnel plots and Egger's linear regression test to assess publication bias [20]. All statistical analyses were carried out using STATA version 11.0 (Stata Corporation College Station, TX, USA).

\section{AUTHOR CONTRIBUTIONS}

JWP, QW and HM conceived and designed the experiments. JWP, HLZ and HM performed publication searches and selection. JWP, QW and HLZ analyzed the data. JWP, GZL, XDS and QW prepared the figures. JWP contributed materials/analysis tools. JWP and WDL wrote and revised the paper. All authors reviewed the manuscript.

\section{ACKNOWLEDGMENTS}

We appreciate the valuable comments from other members of our laboratory.

\section{CONFLICTS OF INTEREST}

The authors declare that they have no conflicts of interest.

\section{FUNDING}

This work was financially supported by the Subitem of the Important and Special Project of the Science and Technology Ministry of China (No. 2018ZX10734404), the National Natural Science Foundation of China (No. 81972949), the Scientific and Technological Innovation Projects of Medicine and Health of Chinese Academy of Medical Sciences (No. 2016-I2M-3-021), the Nanjing Incubation Program for National Clinical Research Center (No. 2019060001), the National Science and Technology Infrastructure of China (No. NPRC-32) and the National Mega-project for Innovative Drugs (No. 2019ZX09721001).

\section{REFERENCES}

1. Stone JH, Frigault MJ, Serling-Boyd NJ, Fernandes AD, Harvey L, Foulkes AS, Horick NK, Healy BC, Shah R, Bensaci AM, Woolley AE, Nikiforow S, Lin N, et al, and BACC Bay Tocilizumab Trial Investigators. Efficacy of tocilizumab in patients hospitalized with covid-19. N Engl J Med. 2020; 383:2333-44. https://doi.org/10.1056/NEJMoa2028836 PMID:33085857

2. Antinori S, Galimberti L, Milazzo L, Ridolfo AL. Bacterial and fungal infections among patients with SARS-CoV-2 pneumonia. Infez Med. 2020; 28:29-36. PMID:32532935
3. Jiang $\mathrm{W}$, Li W, Xiong $\mathrm{L}, \mathrm{Wu} \mathrm{Q}, \mathrm{Wu}$ J, He B, Shen J, Pang R, Luo T, Guo Y, Yang Y, Han Y, Dai W, et al. Clinical efficacy of convalescent plasma therapy on treating COVID-19 patients: Evidence from matched study and a meta-analysis. Clin Transl Med. 2020; 10:e259. https://doi.org/10.1002/ctm2.259 PMID:33377664

4. Wu F, Zhou Y, Wang Z, Xie M, Shi Z, Tang Z, Li X, Li X, Lei C, Li Y, Ni Z, Hu Y, Liu X, et al, and Medical Treatment Expert Group for COPD and COVID-19. Clinical characteristics of COVID-19 infection in chronic obstructive pulmonary disease: a multicenter, retrospective, observational study. J Thorac Dis. 2020; 12:1811-23. https://doi.org/10.21037/jtd-20-1914 PMID:32642086

5. Yang $X, Y u ~ Y, X u$ J, Shu $H$, Xia J, Liu H, Wu Y, Zhang L, Yu $Z$, Fang $M, Y u T$, Wang $Y$, Pan $S$, et al. Clinical course and outcomes of critically ill patients with SARS-CoV-2 pneumonia in Wuhan, China: a single-centered, retrospective, observational study. Lancet Respir Med. 2020; 8:475-81.

https://doi.org/10.1016/S2213-2600(20)30079-5 PMID:32105632

6. Nasir N, Farooqi J, Mahmood SF, Jabeen K. COVID-19associated pulmonary aspergillosis (CAPA) in patients admitted with severe COVID-19 pneumonia: An observational study from Pakistan. Mycoses. 2020; 63:766-70.

https://doi.org/10.1111/myc.13135 PMID:32585069

7. Hughes S, Troise O, Donaldson H, Mughal N, Moore LS. Bacterial and fungal coinfection among hospitalized patients with COVID-19: a retrospective cohort study in a UK secondary-care setting. Clin Microbiol Infect. 2020; 26:1395-99.

https://doi.org/10.1016/i.cmi.2020.06.025

PMID:32603803

8. Salehi M, Ahmadikia K, Mahmoudi S, Kalantari S, Jamalimoghadamsiahkali S, Izadi A, Kord M, Dehghan Manshadi SA, Seifi A, Ghiasvand F, Khajavirad N, Ebrahimi S, Koohfar A, et al. Oropharyngeal candidiasis in hospitalised COVID-19 patients from Iran: species identification and antifungal susceptibility pattern. Mycoses. 2020; 63:771-78. https://doi.org/10.1111/myc.13137 PMID: 32609906

9. Dong X, Cao YY, Lu XX, Zhang JJ, Du H, Yan YQ, Akdis CA, Gao YD. Eleven faces of coronavirus disease 2019. Allergy. 2020; 75:1699-709. https://doi.org/10.1111/all.14289 PMID:32196678

10. Zhu X, Ge Y, Wu T, Zhao K, Chen Y, Wu B, Zhu F, Zhu B, Cui L. Co-infection with respiratory pathogens among COVID-2019 cases. Virus Res. 2020; 285:198005. https://doi.org/10.1016/i.virusres.2020.198005 PMID: $\underline{32408156}$ 
11. White PL, Dhillon R, Cordey A, Hughes H, Faggian F, Soni S, Pandey M, Whitaker $\mathrm{H}$, May A, Morgan M, Wise MP, Healy B, Blyth I, et al. A national strategy to diagnose COVID-19 associated invasive fungal disease in the ICU. Clin Infect Dis. 2020. [Epub ahead of print]. https://doi.org/10.1093/cid/ciaa1298 PMID:32860682

12. Rawson TM, Moore LS, Zhu N, Ranganathan N, Skolimowska K, Gilchrist M, Satta G, Cooke G, Holmes A. Bacterial and fungal coinfection in individuals with coronavirus: a rapid review to support COVID-19 antimicrobial prescribing. Clin Infect Dis. 2020; 71:2459-68.

https://doi.org/10.1093/cid/ciaa530 PMID:32358954

13. Wang Z, Yang B, Li Q, Wen L, Zhang R. Clinical features of 69 cases with coronavirus disease 2019 in Wuhan, China. Clin Infect Dis. 2020; 71:769-77. https://doi.org/10.1093/cid/ciaa272 PMID:32176772

14. Zuo T, Zhan H, Zhang F, Liu Q, Tso EY, Lui GC, Chen N, Li A, Lu W, Chan FK, Chan PK, Ng SC. Alterations in fecal fungal microbiome of patients with COVID-19 during time of hospitalization until discharge. Gastroenterology. 2020; 159:1302-10.e5. https://doi.org/10.1053/i.gastro.2020.06.048 PMID:32598884

15. Lansbury L, Lim B, Baskaran V, Lim WS. Co-infections in people with COVID-19: a systematic review and metaanalysis. J Infect. 2020; 81:266-75. https://doi.org/10.1016/j.jinf.2020.05.046 PMID:32473235

16. Garcia-Vidal C, Sanjuan G, Moreno-García E, PuertaAlcalde P, Garcia-Pouton N, Chumbita M, FernandezPittol M, Pitart C, Inciarte A, Bodro M, Morata L, Ambrosioni J, Grafia I, et al, and COVID-19 Researchers
Group. Incidence of co-infections and superinfections in hospitalized patients with COVID-19: a retrospective cohort study. Clin Microbiol Infect. 2021; 27:83-88.

https://doi.org/10.1016/i.cmi.2020.07.041 PMID:32745596

17. Falces-Romero I, Ruiz-Bastián $M$, Díaz-Pollán $B$, Maseda E, García-Rodríguez J, and SARS-CoV-2 Working Group. Isolation of aspergillus spp. In respiratory samples of patients with COVID-19 in a Spanish tertiary care hospital. Mycoses. 2020; 10:1111. https://doi.org/10.1111/myc.13155

PMID:32749040

18. Álvarez Fernández $M$, Armando Meléndez $D$, Aymerich de Franceschi M. COVID-19 associated pulmonary aspergillosis. Med Intensiva. 2020; S0210-5691: 30277-71.

https://doi.org/10.1016/i.medin.2020.07.014 PMID:33077243

19. Chen N, Zhou M, Dong X, Qu J, Gong F, Han Y, Qiu Y, Wang J, Liu Y, Wei Y, Xia J, Yu T, Zhang X, Zhang L. Epidemiological and clinical characteristics of 99 cases of 2019 novel coronavirus pneumonia in Wuhan, China: a descriptive study. Lancet. 2020; 395:507-13. https://doi.org/10.1016/S0140-6736(20)30211-7 PMID:32007143

20. Jiang W, Sun H, Zhang J, Zhou Q, Wu Q, Li T, Zhang C, Li $\mathrm{W}$, Zhang $\mathrm{M}$, Xia X. Polymorphisms in protamine 1 and protamine 2 predict the risk of male infertility: a metaanalysis. Sci Rep. 2015; 5:15300.

https://doi.org/10.1038/srep15300

PMID:26472740 


\section{SUPPLEMENTARY MATERIALS}

\section{Supplementary Figures}

A

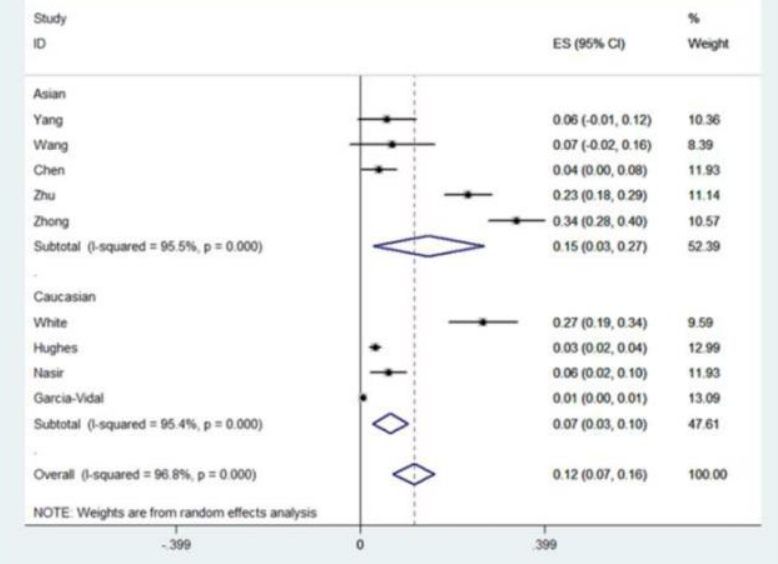

C

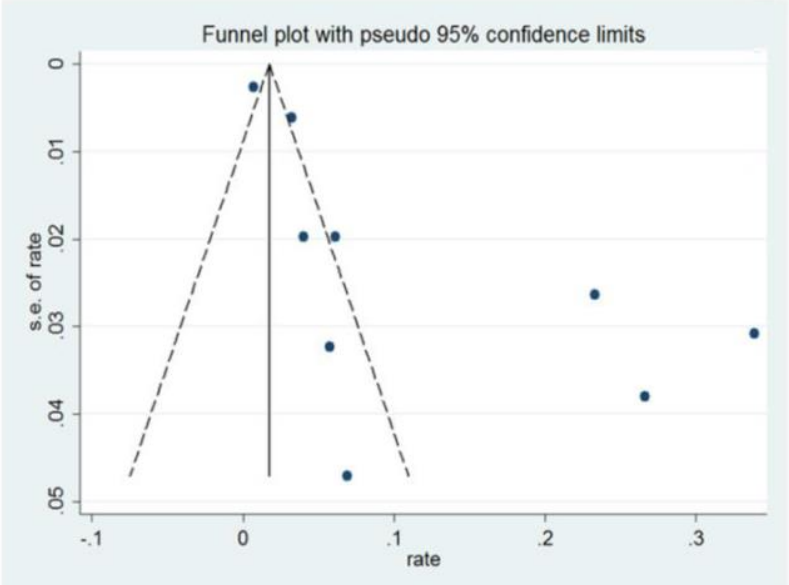

B

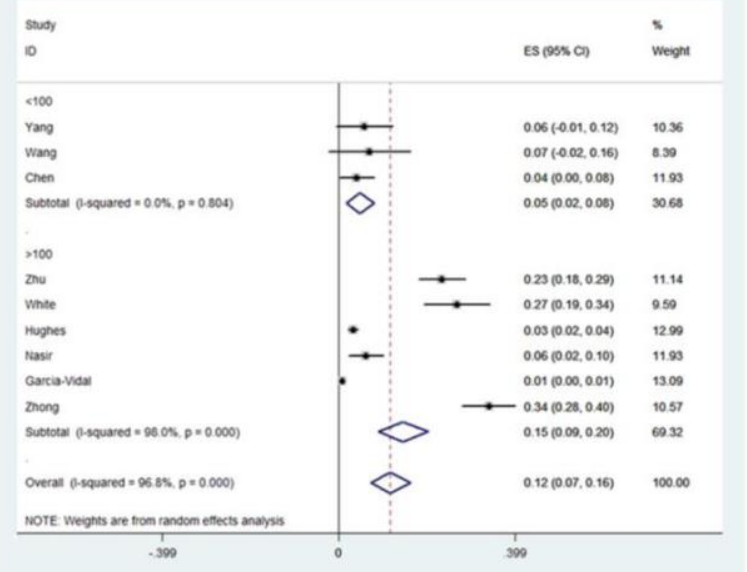

D

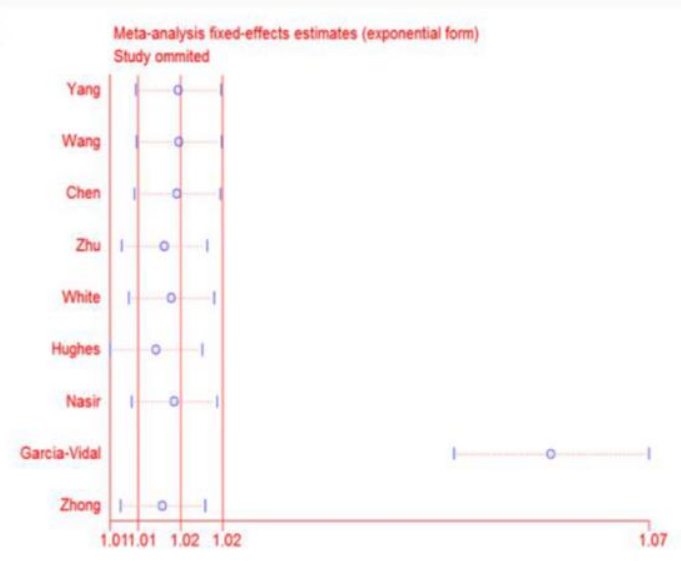

Supplementary Figure 1. The association of fungal infection in COVID-19 patients. (A, B) Forest plot of fungal infection in COVID-19 patients (A) Race subgroup analysis (B) Case size subgroup analysis (C) The funnel plot of fungal infection in COVID-19 patients (D) Sensitivity analysis of fungal infection in COVID-19 patients. 
A

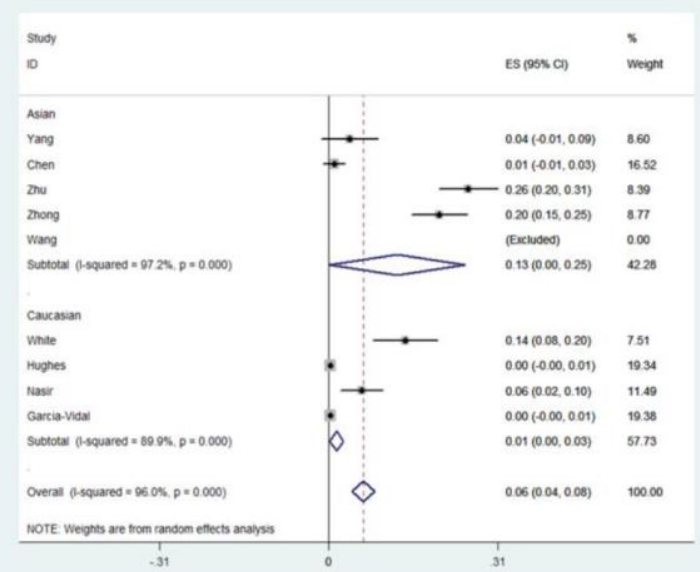

C

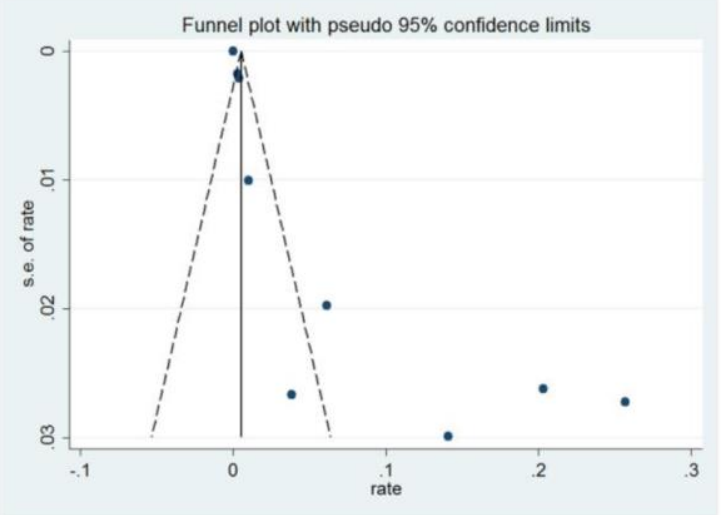

B

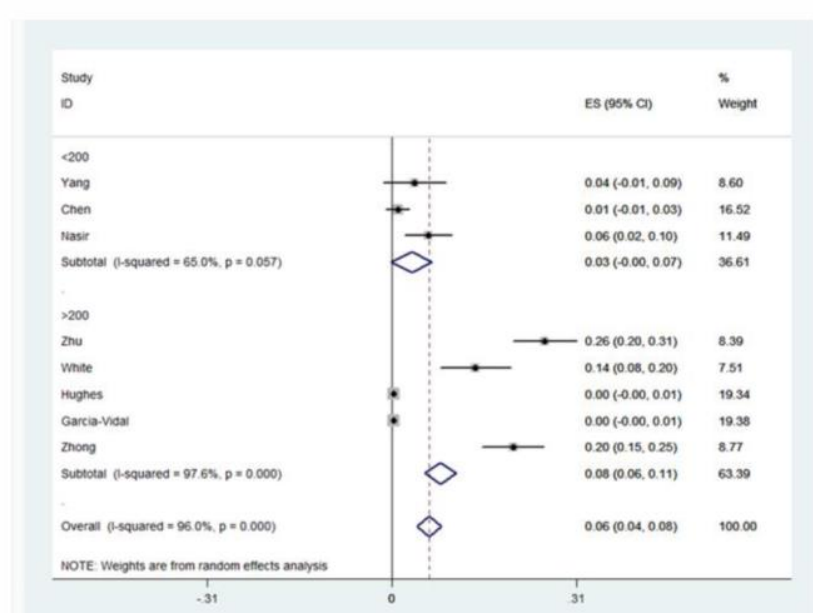

D

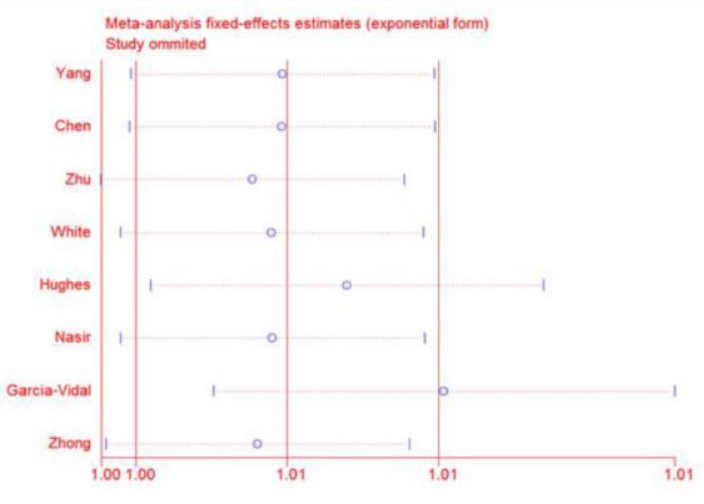

Supplementary Figure 2. The association of aspergillus infection in COVID-19 patients. (A, B) Forest plot of aspergillus infection in COVID-19 patients (A) Race subgroup analysis (B) Case size subgroup analysis (C) The funnel plot of aspergillus infection in COVID-19 patients (D) Sensitivity analysis of aspergillus infection in COVID-19 patients. 
A

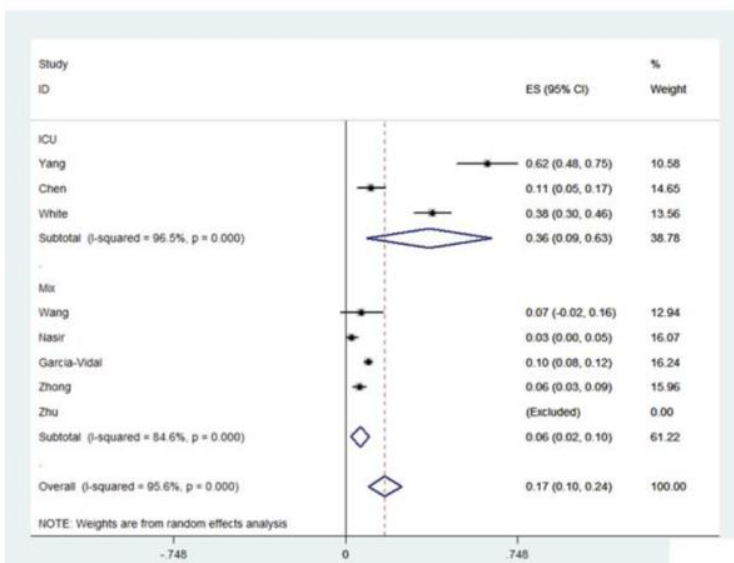

C

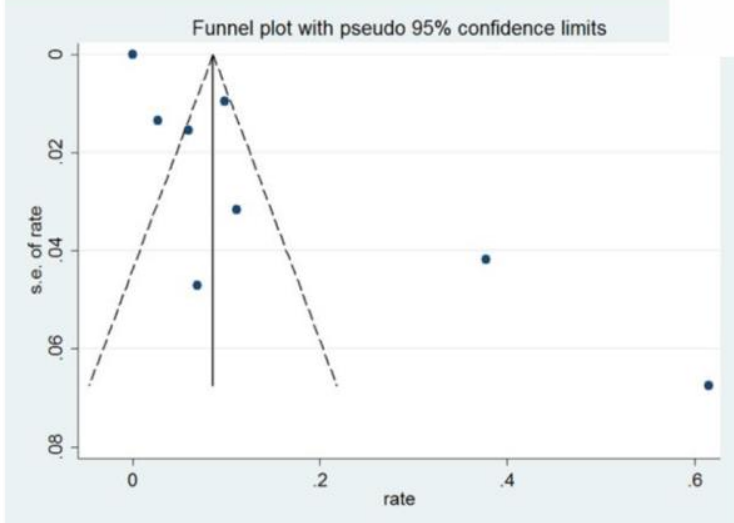

B

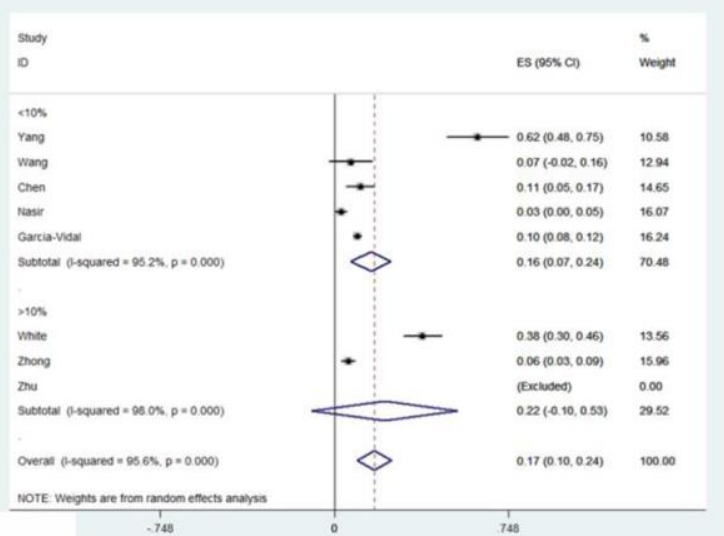

D

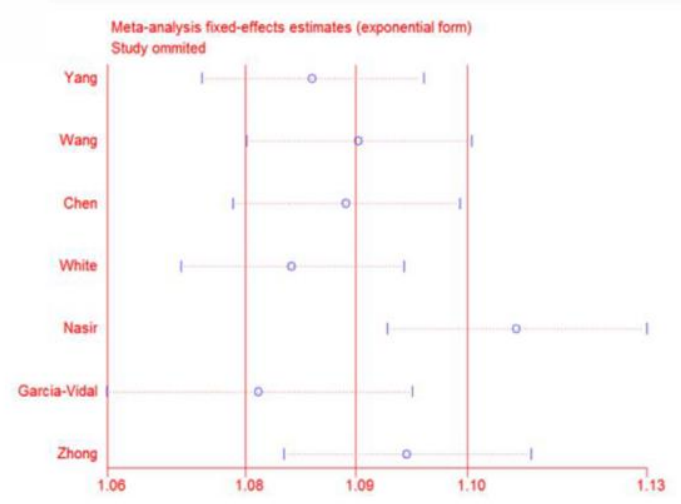

Supplementary Figure 3. The association of mortality in fungal co-infections and COVID-19 patients. (A, B) Forest plot of mortality in fungal co-infections and COVID-19 patients (A) Severity subgroup analysis (B) Case size subgroup analysis (C) The funnel plot of mortality in fungal co-infections and COVID-19 patients (D) Sensitivity analysis of mortality in fungal co-infections and COVID-19 patients. 